\title{
Chyle Leak after a Simple Hemithyroidectomy: An Extremely Rare Situation
}

Adi S Muhammed ${ }^{a}$, Shahrun N Suhaimi ${ }^{a}$, Suraya Othman ${ }^{b}$, Nor Faezan A Rashid ${ }^{b}$, Rohaizak Muhammad ${ }^{a}$ ${ }^{a}$ Department of Surgery, UKM Medical Centre, Hospital Canselor Tuanku Muhriz, Malaysia

${ }^{b}$ Department of Surgery, Universiti Teknologi MARA, Malaysia

\section{ABSTRACT}

Chyle leak is a well-known complication that can occur after a thyroidectomy with neck dissection; however, it rarely occurs after thyroidectomy alone. Here, we report a case of chyle leak following a left hemithyroidectomy for a benign solitary thyroid nodule. Based on the literature search, this is only the second reported case of a chyle leak after a simple hemithyroidectomy without any central or lateral neck dissection. Amongst the possible causes, we hypothesize that the use of intraoperative nerve monitor may be a contributing factor. Treatment options are explored and the patient was treated successfully with nonoperative management.

KEYWORDS: chyle leak, thyroidectomy, intraoperative nerve monitor, neck dissection, thoracic duct, anatomical variants

\section{INTRODUCTION}

Thyroidectomy is a common surgery performed for both benign and malignant thyroid conditions. Several complications may arise from surgical injuries to the related anatomical structures within the neck, such as the parathyroid glands and recurrent laryngeal nerve (RLN). Hypocalcaemia and RLN injuries are the two most commonly encountered problems postoperatively. However, the most feared complication of thyroid surgery is a sudden neck hematoma, which can be life threatening. Other less common complications include dysphagia, seroma, Horner's syndrome and chyle leak. ${ }^{1}$ Although rare, chyle leaks can lead to serious problems, such as chylothorax, hypovolaemia, electrolyte imbalance, nutritional deficiency and immunosuppression. ${ }^{2}$

\section{CASE}

A 65-year-old woman presented with left thyroid swelling that remained stable over five years. She

Corresponding Author:

Assoc. Prof. Dr. Shahrun Niza Abdullah Suhaimi

Consultant, Endocrine and Breast Surgeon

Department of Surgery,

UKM Medical Centre,

Jalan Yaacob Latiff, 56000 Cheras,

Kuala Lumpur, Malaysia.

Tel No: +6039145 555

Email: shahrun72.sn@gmail.com was euthyroid, and there were neither compressive symptoms nor voice changes. The examination revealed a $3-\mathrm{cm}$ well-defined left solitary thyroid nodule, with no palpable cervical node. Her thyroid function test was normal and the ultrasound of the thyroid revealed a spongiform nodule with halo sign.

The fine-needle aspiration cytology revealed a benign lesion.

This patient underwent an open left hemithyroidectomy through a $4-\mathrm{cm}$ collar neck incision. It was a straightforward procedure without any obvious complications. Continuous intraoperative nerve monitoring was used for the identification and preservation of the RLN. The parathyroid glands were also identified and preserved. Valsalva manoeuvre was performed prior to closure and showed no bleeding or air leaks. Finally, a $14 \mathrm{Fr}$ vacuum drain was inserted, and the surgery was completed in 45 minutes.

On postsurgical day two, milky fluid was seen coming from the drain, which showed elevated serum triglycerides $(7 \mathrm{~g} / \mathrm{dl})$. Diagnosis of chyle leak was made, and the patient was started on a low fat and medium chain triglycerides diet. Since the chyle fistula had a low output ( 5 to $10 \mathrm{ml} /$ day), the drain was removed on day four, and a compression dressing was applied. The patient was discharged on 
postsurgical day five. When she came in for a follow -up one week later, there was a fluctuant swelling measuring $5 \times 5 \mathrm{~cm}$ over the previous wound (Figure 1).

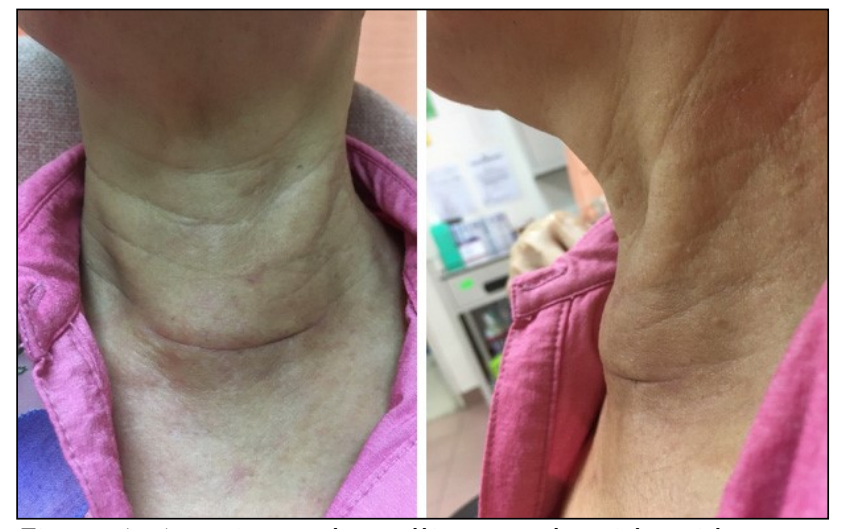

Figure 1: Anterior neck swelling post hemithyroidectomy secondary to chyloma (anterior and lateral view)

Upon needle aspiration, $30 \mathrm{ml}$ of chyle was evacuated (Figure 2). This was repeated weekly over the course of one month before it was completely resolved. The final histopathology reported nodular hyperplasia with degenerative changes.

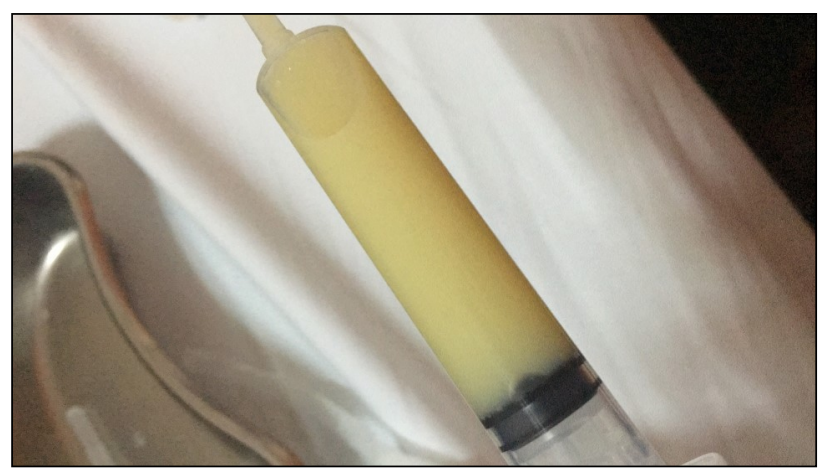

Figure 2: Milky fluid aspirate from patient's neck wound, typical of chyle

\section{DISCUSSION}

Chyle leak is the result of an injury to the lymphatic channels, of which the main one is the thoracic duct (TD). It is usually associated with head and neck or thyroid surgery for malignant conditions in which neck dissection is performed, especially on the left side. However, right-sided lymphatic injuries also occur in up to $25 \%$ of all chyle leaks. ${ }^{2}$ Overall, the incidence of chyle leak is low (usually $<1 \%$ ), but it can reach up to $8.3 \% .^{3}$ Higher incidence is seen in more extensive neck dissection $(1.4 \%$ with central neck dissection alone; $8.3 \%$ with lateral neck dissection). ${ }^{4}$ The risk is also expectedly higher in redo surgery. This is only the second reported case of a chyle leak after a simple hemithyroidectomy after Madnani. ${ }^{5}$

Chyle leak can be identified intraoperatively or postoperatively. However, an intraoperative identification is difficult because the lymphatic fluid is usually clear and sparse, since patients are usually fasted before surgery. Increasing the intrathoracic pressure with Valsalva manoeuvre may help to increase the lymphatic flow and in identification. ${ }^{2}$ If it is recognized intraoperatively, an immediate repair or ligation of the injured duct should be performed. More commonly, chyle leak is detected postoperatively, and the patient may present with localized collection over the wound or a persistent drain output. The patient can also present with a more severe chylothorax. Identification of milky fluid in drain or upon needle aspiration is usually pathognomonic. A laboratory confirmation can be done by measuring the triglyceride level of the fluid, and a level above $100 \mathrm{mg} / \mathrm{dl}$ or the presence of chylomicrons is diagnostic of chyle. ${ }^{2}$

Anatomically, thoracic duct (TD) arises from cisterna chyli within the abdomen and ascends along the thoracic vertebrae, crossing from right to left at the level of the fifth or sixth thoracic vertebra. At the cervical region, it usually curves back before terminating into one of the major veins near the jugulovenous angle. ${ }^{6}$ With regard to surface marking, this covers an area of about $4.4 \mathrm{~cm}^{2}$ in the left supraclavicular fossa, with the lymphovenous communication usually located approximately 4.5 $\mathrm{cm}$ lateral to the midline and $3.0 \mathrm{~cm}$ superior to the sternal notch. ${ }^{7}$

There are several anatomical variations of the TD that have been found in cadavers that can explain the chyle leak that occurred in our case ${ }^{8}$ First, the thoracic duct arch ascends and curves backward into a vein (Figure 3), and typically runs $2-3 \mathrm{~cm}$ above the clavicle, but this can go as high as $5 \mathrm{~cm} .{ }^{6}$ The high arching TD is predisposed to injury during a simple thyroidectomy, even when the dissection is away from the TD region. Second, the TD most commonly ends within $1-2 \mathrm{~cm}$ of the junction between the left subclavian vein and internal jugular vein (IJV) (jugulovenous angle). However, it may have multiple terminal branches and may end in several alternative veins within the neck region, namely the external jugular vein, vertebral vein, 
transverse cervical vein, brachiocephalic vein and suprascapular vein (Figure 3$)^{6}{ }^{6}$

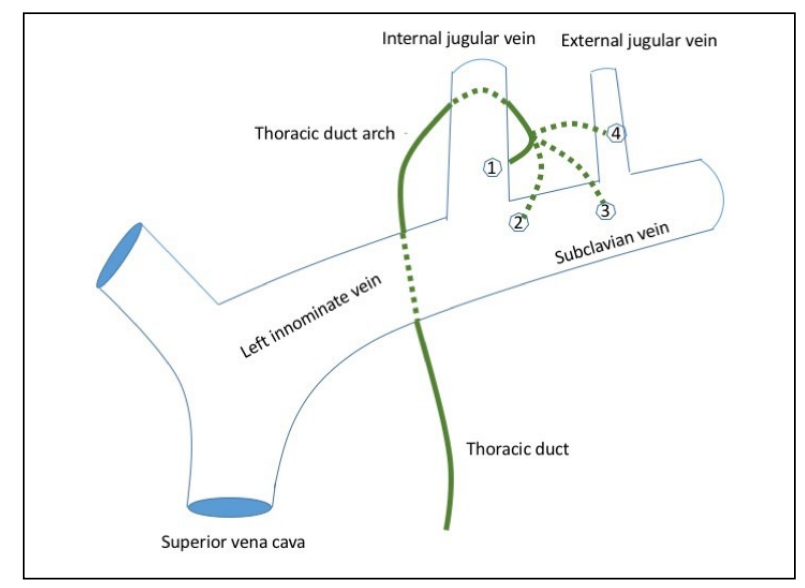

Figure 3: Schematic diagram showing thoracic duct arch and its terminal branches into alternative veins

At the IJV itself, the terminal branches may have multiple entry points into its anterior, posterior or lateral wall (Figure 4). ${ }^{8}$ These complex and thinwalled branches of the TD make them more vulnerable to injury when stretched during the mobilization of the thyroid gland and while searching for the RLN.

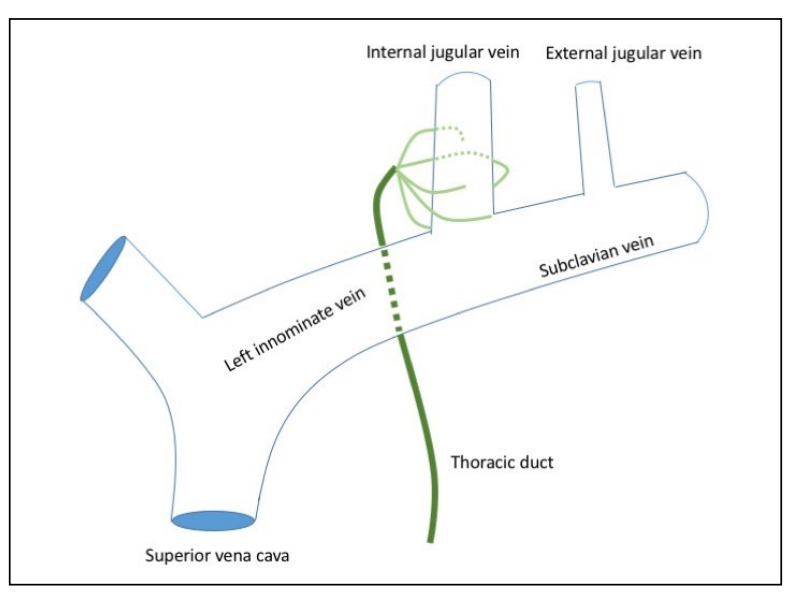

Figure 4: Schematic diagram representing thoracic duct branches entering IJV at different angles (anterior, posterior, medial, lateral and at jugulovenous angles)

We postulated that the use of intraoperative nerve monitoring (IONM) may be a contributing factor. IONM requires an open dissection of the carotid sheath in order to locate the vagus nerve. This nerve lies posterior to and in between the common carotid artery and IJV. It requires skeletonization to allow the placement of the conduction wire. During this process, an injury to abnormally situated lymphatic branches can potentially cause chyle leak. Nonetheless, there has been no other documentation or reports of such incidence.
A continuous leak may lead to the formation of a chylous fistula. An injury to the main $T D$ is commonly associated with a high output fistula ( $>500$ $\mathrm{ml} /$ day), while an injury to the small branches may cause a low output fistula ( $<500 \mathrm{ml} /$ day). While a low output fistula usually responds to a conservative approach, a high output fistula is more difficult to manage and usually requires a surgical intervention. ${ }^{9}$ In the majority of cases, conservative management is the preferred initial approach. Apart from repeated needle aspiration and a compression dressing, other approaches that have been proposed include bed rest, head elevation, avoiding conditions that raise the intra-abdominal pressure (such as constipation) and diet control via a strict non-fat, low-fat or medium chain fatty acid diet. ${ }^{2}$ These aim to reduce the lymphatic flow amount and promote healing of the injured duct. If all else fails, the patient may be kept completely nil by mouth and administered total parenteral nutrition if prolonged fasting is expected.

Somatostatin or its analogue, octreotide, has been used as an adjunct to help reduce chyle production by reducing the gastric, pancreatic and intestinal secretions. $^{2}$ A conservative strategy is usually effective in $58 \%$ to $100 \%$ of the cases. ${ }^{3}$

Surgical intervention is usually reserved for those cases in which the conservative treatment fails. This can be done with an open surgical exploration or through the endolymphatic route. Trans-lymphatic embolization with sclerosing agents, such as OK-432 or tetracycline, requires a highly experienced interventional radiologist.

Surgical exploration is generally recommended for a high output fistula that persists for four to five days, despite the best conservative approach. Identification of the site of the leak may be difficult due to adhesions and inflammation, and performing the Valsalva manoeuvre or having the patient ingest fatty foods before surgery, may help in the localization. The leak site can be ligated, covered with a sternocleidomastoid muscle flap or sealed using sclerosing or adhesive agents or mesh, followed by suction drainage.

Finally, thoracoscopic ligation of the TD through the right chest is the salvage procedure of choice if everything else fails. ${ }^{2}$ 


\section{CONCLUSION}

Chyle leak following thyroidectomy is an extremely rare complication. Apart from neck dissection, anatomical variations of the thoracic duct may predispose it to injury. The use of intraoperative nerve monitoring may cause adverse effects such as chyle leak. Therefore, surgeons should first try to avoid such complications by appreciating the anatomy of the TD as well as its variations. Moreover, surgeons should be familiar with the multiple treatment options that are available and the stepwise approach in its management so that patients have the best chance for complete resolution.

\section{DECLARATION OF CONTRIBUTION}

This manuscript is the original work with considerable contributions from all authors. With the submission of this manuscript, we would like to undertake that:

I. All authors have directly participated and made substantial contribution each to qualify for authorship according to CMJ authorship criteria.

II. All authors have read and approved the final version of the submitted manuscript to its publication in this form.

III. This manuscript has not been published previously, nor currently under consideration for publication elsewhere.

IV. This manuscript will not be copyrighted, submitted or published elsewhere while acceptance by your journal is under consideration.

v. There are no other directly related manuscripts published by any authors of this case report.

VI. There is nothing for disclosure and no conflicts of interest to declare pertaining this article.

VII. There is no breach in patient privacy, the codes of ethics in medical researchhave been complied with and patient's informed consent has been obtained.

VIII. My institution's (UKM Medical Centre) authority is fully aware of this submission.

\section{AFFILIATIONS}

None

\section{ABBREVIATIONS}

RLN Recurrent laryngeal nerve

TD Thoracic duct

IJV Internal jugular vein

IONM Intraoperative nerve monitoring

\section{REFERENCES}

1. Christou N, Mathonnet M. Complications after total thyroidectomy. J Visc Surg. 2013 Sep;150(4):249-56.

2. Delaney SW, Shi H, Shokrani A, Sinha UK. Management of Chyle Leak after Head and Neck Surgery: Review of Current Treatment Strategies. Int J Otolaryngol. 2017;2017:112.

3. Rammal A, Zawawi F, Varshney R, Hier MP, Payne RJ, Mlynarek AM. Chyle leak: A rare complication post-hemithyroidectomy. Case report and review of literature. Otolaryngol Pol. 2014 Jul;68(4):204-7.

4. Roh J-L, Yoon Y-H, Park Cl. Chyle leakage in patients undergoing thyroidectomy plus central neck dissection for differentiated papillary thyroid carcinoma. Ann Surg Oncol. 2008 Sep;15(9):2576-80.

5. Madnani D, Myssiorek D. Left cervical chyloma following right thyroidectomy. Ear Nose Throat J. 2003 Jul;82(7):522-4.

6. Phang K, Bowman M, Phillips A, Windsor J. Review of thoracic duct anatomical variations and clinical implications: TD Anatomical Variations and Clinical Implications. Clin Anat. 2014 May;27(4):63744.

7. Ammar K, Tubbs RS, Smyth MD, Wellons JC, Blount JP, Salter G, et al. Anatomic landmarks for the cervical portion of the thoracic duct. Neurosurgery. 2003 Dec;53 (6):1385-1387; discussion 1387-1388.

8. Gottlieb MI, Greenfield J. Variations in the terminal portion of the human thoracic duct. AMA Arch Surg. 1956 Dec;73(6):955-9.

9. Lee YS, Kim B-W, Chang H-S, Park CS. Factors predisposing to chyle leakage following thyroid cancer surgery without lateral neck dissection. Head Neck. 2013 Aug;35(8):1149-52. 